J. Väisälä, M. Vuorinen and H. Wallin

Nagoya Math. J.

Vol. 135 (1994), 121-148

\title{
THICK SETS AND QUASISYMMETRIC MAPS
}

\author{
JUSSI VÄISÄLÄ, MATTI VUORINEN AND HANS WALLIN
}

\section{Introduction}

1.1. Thickness. Let $E$ be a real inner product space. For a finite sequence of points $a_{0}, \ldots, a_{k}$ in $E$ we let $a_{0} \ldots a_{k}$ denote the convex hull of the set $\left\{a_{0}, \ldots\right.$, $a_{k}$ \}. If these points are affinely independent, the set $\Delta=a_{0} \ldots a_{k}$ is a $k$-simplex with vertices $a_{0}, \ldots, a_{k}$. It has a well-defined $k$-volume written as $m_{k}(\Delta)$ or briefly as $m(\Delta)$. We are interested in sets $A \subset E$ which are "nowhere too flat in dimension $k$ ". More precisely, suppose that $A \subset E, q>0$ and that $k$ is a positive integer. We let $\bar{B}(x, r)$ denote the closed ball with center $x$ and radius $r$. We say that $A$ is $(q, k)$-thick if for each $x \in A$ and $r>0$ such that $A \backslash \bar{B}(x, r) \neq \emptyset$ there is a $k$-simplex $\Delta$ with vertices in $A \cap \bar{B}(x, r)$ such that $m_{k}(\Delta) \geq q r^{k}$.

It is easy to see that the closure $\bar{A}$ of a $(q, k)$-thick set $A$ is $\left(q^{\prime}, k\right)$-thick for each $q^{\prime}<q$. In the case $\operatorname{dim} E<\infty, \bar{A}$ is in fact $(q, k)$-thick. Conversely, if $\bar{A}$ is $(q, k)$-thick, $A$ is $\left(q^{\prime}, k\right)$-thick for all $q^{\prime}<q$. Without essential loss of generality, it is thus sufficient to consider only closed sets $A \subset E$.

We also say that $A$ is $k$-thick if $A$ is $(q, k)$-thick for some $q>0$. It is easy to see that a $p$-thick set is $k$-thick for all $k \leq p$.

1.2. Examples. We consider sets in the Euclidean $n$-space $R^{n}$. A set $A \subset R^{n}$ can be $k$-thick only for $k \leq n$. A $k$-dimensional ball and a $k$-cube are clearly $k$-thick but not $p$-thick for $p>k$. The Cantor middle-third set is 1 -thick. If $A$ is an arc which has a tangent at some point, $A$ is not 2 -thick. In particular, rectifiable arcs are not 2-thick. On the other hand, the Koch snowflake curve in $R^{2}$ is 2 -thick. A $c$-John domain [NV], 2.26, and its closure in $R^{n}$ are $(q, n)$-thick with $q=q(c, n)$.

1.3. Background. Thick sets arise naturally from various questions of analysis. For example, in [Vä3], Th. 6.2, it was proved that if $A$ is compact and

Received June 22, 1992. 
$n$-thick in $R^{n}$, and if $M$ is sufficiently close to 1 , then each $M$-bilipschitz map $f: A \rightarrow R^{n}$ has an $M_{1}$-bilipschitz extension $g: R^{n} \rightarrow R^{n}$, where $M_{1}=M_{1}(M, A)$ $\rightarrow 1$ as $M \rightarrow 1$.

On the other hand, the $n$-thick sets in $R^{n}$ are precisely the sets for which certain polynomial inequalities of Markov type are valid. More precisely, let $\mathscr{P}_{k}$ be the family of all algebraic polynomials of $n$ real variables of total degree at most $k$. Then a closed set $A \subset R^{n}$ is $n$-thick if and only if for each positive integer $k$ there is a constant $c=c(A, n, k)$ such that

$$
\|\operatorname{grad} P\|_{A \cap B} \leq \frac{c}{r}\|P\|_{A \cap B}
$$

for each $P \in \mathscr{P}_{k}$ and for each ball $B=\bar{B}(x, r)$ with $x \in A$ and $r<d(A)$; here $\|f\|_{Q}$ denotes the maximum of $|f(x)|$ over $x \in Q$ and $d(A)$ is the diameter of $A$. This is a similarity invariant version of [WW, Proposition 7]. This condition on $A$, introduced in 1980, is important in the study of polynomial approximation and interpolation and function spaces on $A$; see [JW].

For another example of the idea of thickness, see the recent paper of Rohde [Ro], p. 111. The linear approximation condition in [MV] is in a sense opposite to the thickness condition.

1.4. Summary of results. The purpose of this paper is to study the invariance properties of thick sets. First observe that since the snowflake curve is a quasicircle, the property of 2-thickness is not preserved under quasiconformal maps of the plane. In 6.2 we show that it is not even a bilipschitz invariant. However, we shall show that a $K$-quasiconformal map $f: R^{n} \rightarrow R^{n}$ maps $(q, k)$-thick sets onto $\left(q_{1}, k\right)$-thick sets provided that $K$ is sufficiently small: $K$ $\leq K_{0}(q, k)$. Moreover, $q_{1}$ depends only on $(q, K, k)$, and $q_{1} \rightarrow q$ as $K \rightarrow 1$.

A $K$-quasiconformal map $f: R^{n} \rightarrow R^{n}$ is $s$-quasisymmetric with $s=s(K, n)$ $\rightarrow 0$ as $K \rightarrow 1$. For this result and for the definition of quasisymmetry, see Section 2. We shall obtain the above result on quasiconformal invariance as a corollary of a more general result on $s$-quasisymmetric maps $f: A \rightarrow E^{\prime}$ where $A$ is a closed set in an inner product space $E$ and $E^{\prime}$ is another inner procuct space. More precisely, we show in Section 4 that if $A$ is $(q, k)$-thick and $s$ is less than a number $s_{0}=s_{0}(q, k)$, then $f A$ is $\left(q_{1}, k\right)$-thick with $q_{1}=q_{1}(q, s, k) \rightarrow q$ as $s \rightarrow 0$.

Bilipschitz versions are given in Section 6 . In Section 5 we consider the special case where $A$ is an arc. For example, we show that if $A$ is the image of a line segment under an $s$-quasisymmetric map, then for all $k$ there is $q=q(s, k)$ such that $A$ is not $(q, k)$-thick. Moreover, $q \rightarrow 0$ as $s \rightarrow 0$. 
Various methods based on normal families are often used in the study of $K$-quasiconformal, $K$-quasiregular and $s$-quasisymmetric maps. While such methods provide a convenient technique for studying qualitative properties of these maps, they fail to give explicit estimates. Our goal is to give, whenever possible, explicit bounds that are still significant as $K \rightarrow 1$ or $s \rightarrow 0$. The bounds obtained are presumably not always very sharp. In some cases we also estimate the sharpness of the bounds by examples.

Our methods are rather elementary in the quasisymmetric and bilipschitz cases. For quasiconformal maps, an essential tool is $[\mathrm{Vu}]$, Theorem 1.8 , which gives explicit bounds for the distortion of $K$-quasiconformal maps of $R^{n}$ with small $K$.

1.5. Notation. Throughout the paper, we assume that $E$ is an inner product space with norm $|x|=(x \cdot x)^{1 / 2}$. We let $R^{n}$ denote the euclidean $n$-space with the usual inner product and the standard basis $e_{1}, \ldots, e_{n}$. For two nonempty sets $A, B$ $\subset E$, we let $d(A, B)$ denote the distance between $A$ and $B$, and $d(A)$ is the diameter of $A$. Open and closed balls with center $x$ and radius $r$ are written as $B(x, r)$ and $\bar{B}(x, r)$, respectively, and $S(x, r)$ is the sphere $\partial B(x, r)$.

\section{Quasisymmetry and quasiconformality}

In this section we give preliminary results on the relations between $s$-quasisymmetric and $K$-quasiconformal maps of $R^{n}$. Particular emphasis will be on the case where $s \rightarrow 0$ or, equivalently, $K \rightarrow 1$.

2.1. Definition. Let $\eta:[0, \infty) \rightarrow[0, \infty)$ be a homeomorphism. An injective map $f: X \rightarrow Y$ between metric spaces is $\eta$-quasisymmetric or $\eta-\mathrm{QS}$ if

$$
\frac{|f a-f x|}{|f b-f x|} \leq \eta\left(\frac{|a-x|}{|b-x|}\right)
$$

for each triple $x, a, b \in X$ with $b \neq x$. It follows that for distinct points $x, a, b$ $\in X$ we have

$$
\frac{1}{\eta\left(\frac{|b-x|}{|a-x|}\right)} \leq \frac{|f a-f x|}{|f b-f x|} \leq \eta\left(\frac{|a-x|}{|b-x|}\right)
$$

Moreover, the inverse map $f^{-1}: f X \rightarrow X$ is $\eta^{\prime}$-QS with $\eta^{\prime}(t)=\eta^{-1}\left(t^{-1}\right)^{-1}$. 
2.2. Definition. Let $s \geq 0$. A map $f: X \rightarrow Y$ is $s$-QS if $f$ is $\eta-Q S$ in the sense of 2.1 for some $\eta$ such that

$$
\eta(t) \leq t+s
$$

for all $t \in(0, \max \{1,1 / s\})$.

2.3. Remark. The $\eta-Q S$ maps were introduced in $\left[\mathrm{TV}_{1}\right]$. The notion of $s$-quasisymmetry is from $\left[\mathrm{TV}_{2}\right]$. For values $s>1$ our definition of $s-\mathrm{QS}$ maps differs from the earlier definition. A map $f: X \rightarrow Y$ is $0-\mathrm{QS}$ if and only if it is a similarity: there is $\lambda>0$ such that $|f x-f y|=\lambda|x-y|$ for all $x, y \in X$.

2.4. Relations between $\mathbf{Q C}$ and $\mathbf{Q S}$ maps. In the rest of this section we shall only consider homeomorphisms between domains $G$ and $G^{\prime}$ in $R^{n}, n \geq 2$. We adopt the definition of a $K$-quasiconformal or a $K$-QC map from [Vä 1 ], based on the moduli of path families.

2.5. Theorem. If $f: G \rightarrow G^{\prime}$ is an $s$-QS homeomorphism, then $f$ is $K$-QC with $K=(1+s)^{n-1}$.

Proof. Fix $x \in G$ and $r \in(0, d(x, \partial G))$. For $a, b \in S(x, r)$ we have

$$
\frac{|f a-f x|}{|f b-f x|} \leq \eta\left(\frac{|a-x|}{|b-x|}\right)=\eta(1) \leq 1+s .
$$

This inequality implies that the linear dilatation of $f$ at $x$ has the upper bound $1+s$, and the theorem follows from the metric definition of quasiconformality [Vä1], 34.2.

Thus QS implies QC. The converse is false in general but true for certain domains, in particular, for maps of the whole space $R^{n}$. We give a quantitative form of this fact in Theorem 2.13. It gives an explicit bound for the case $K \rightarrow 1$; the proof of the earlier result $\left[\mathrm{TV}_{2}\right]$, Th. 2.6 was based on a normal family argument.

The next result is Theorem 1.8 from $[\mathrm{Vu}]$. The function $\varphi_{K, n}:[0,1] \rightarrow$ $[0,1]$ in 2.6 is an increasing homeomorphism, for which several estimates were recently proved in [AVV]. It is crucial for our later applications that the inequalities in 2.6 (1) and 2.6 (4) are in some sense asymptotically sharp as $K \rightarrow 1$. Note that the inequalities in 2.6 (4) could be further refined in view of the results in [AVV]. 
2.6. THEOREM. Let $f: R^{n} \rightarrow R^{n}$ be $K-\mathrm{QC}$. Then $f$ is $\eta_{0}-\mathrm{QS}$ with

(1) $\eta_{0}(1)=\exp \left[6(K+1)^{2} \sqrt{K-1}\right]$,

(2) $\eta_{0}(t)=\eta_{0}(1) \varphi_{K, n}(t), 0<t \leq 1$,

(3) $\eta_{0}(t)=\eta_{0}(1) / \varphi_{1 / K, n}(1 / t), t \geq 1$.

Furthermore, with $\alpha=K^{1 /(1-n)}=1 / \beta$ we have for $0 \leq r \leq 1$ :

(4) $2^{1-K} K^{-K} r^{\beta} \leq \varphi_{1 / K, n}(r) \leq r^{\beta} \leq r \leq r^{\alpha} \leq \varphi_{K, n}(r) \leq 2^{1-1 / K} K r^{\alpha}$.

2.7. THEOREM. A $K$-QC map $f: R^{n} \rightarrow R^{n}$ is $\eta$-QS with $\eta(t)=\phi(K)$. $\max \left\{t^{K}, t^{1 / K}\right\}$ where $\phi(K)=\exp \left[7(K+1)^{2} \sqrt{K-1}\right]$.

Proof. Write $c(K)=\exp \left[6(K+1)^{2} \sqrt{K-1}\right]$. Applying 2.6 and the inequalities $\alpha \geq 1 / K, 2<e, K \leq e^{K-1}$ we get for $0 \leq t \leq 1$ :

$$
\eta_{0}(t) \leq c(K) 2^{1-1 / K} K t^{\alpha} \leq c(K) \exp (K-1 / K) t^{1 / K} \leq \phi(K) t^{1 / K} .
$$

For $t \geq 1$ we use 2.6 and the inequalities $\beta \leq K, \log K \leq K-1$ to obtain

$$
\eta_{0}(t) \leq c(K) 2^{K-1} K^{K} t^{K} \leq c(K) \exp (K-1+K \log K) t^{K} \leq \phi(K) t^{K} .
$$

2.8. LEMma. The function $\eta(t)-t$ is increasing in $t$, where $\eta(t)$ is as in 2.7 .

Proof. The continuous function $g(t)=\eta(t)-t$ is differentiable for $t>0$, $t \neq 1$. It is easy to show that $\phi(K) \geq K$. Using elementary calculus we see that $g^{\prime}(t) \geq 0$ for $t>0, t \neq 1$. Hence $g$ is increasing.

For the purpose of convenient reference we record two elementary inequalities:

$$
\begin{gathered}
x^{x} \geq \exp \left(-\frac{2 \sqrt{x}}{e}\right), \quad x>0, \\
\exp \left(\frac{y}{1+y}\right) \leq 1+y, \quad y \geq 0 .
\end{gathered}
$$

2.11. Lemma. Suppose that $1<K \leq 1+10^{-4}$ and that

$$
0 \leq t \leq 1 /(7 \sqrt[4]{K-1})
$$

Then

$$
\eta(t)-t \leq 7 \sqrt[4]{K-1}
$$


where $\eta$ is the function defined in 2.7 .

Proof. Set $u=1 /(7 \sqrt[4]{K-1})$. By Lemma 2.8 it suffices to show that $\eta(u)-u$ $\leq 1 / u$, or equivalently,

$$
\exp \left[7(K+1)^{2} \sqrt{K-1}\right] \leq u^{1-K}\left(1+u^{-2}\right) .
$$

By (2.9) we have

$$
u^{1-K}=(7 \sqrt[4]{K-1})^{K-1} \geq(K-1)^{K-1} \geq \exp \left(-\frac{2}{e} \sqrt{K-1}\right) .
$$

Hence it suffices to show that

$$
\exp \left[\left(\frac{2}{e}+7(K+1)^{2}\right) \sqrt{K-1}\right] \leq 1+49 \sqrt{K-1} .
$$

Writing $y=49 \sqrt{K-1}$ we have $1+y \leq 1+49 / 100<3 / 2$, and thus

$$
\frac{y}{1+y} \geq 30 \sqrt{K-1} \geq\left(\frac{2}{e}+7(K+1)^{2}\right) \sqrt{K-1} .
$$

By (2.10) this implies (2.12).

2.13. THEOREM. A $K-\mathrm{QC}$ map $f: R^{n} \rightarrow R^{n}$ is $s-\mathrm{QS}$ with $s=s(K)$. For $K \leq 1+10^{-4}$ we can choose $s(K)=7 \sqrt[4]{K-1}$.

Proof. The first part of the theorem is well known and follows, for example, from 2.7. The second part follows from 2.7 and 2.11 .

2.14. The case $n=2$. In the 2 -dimensional case, the results above can be refined, since now the function $\eta_{0}$ of 2.6 can be replaced by another function, whose properties are well known. Indeed, a $K-Q C$ map $f: R^{2} \rightarrow R^{2}$ is $\eta-Q S$ with

$$
\eta(t)=\eta_{K, 2}(t)=\frac{u^{2}}{1-u^{2}}, \quad u=\varphi_{K, 2}\left(\sqrt{\frac{r}{1+r}}\right),
$$

where $\varphi_{K, 2}$ is the frequently occurring function in the theory of $\mathrm{QC}$ maps defined in terms of elliptic integrals. See, for example, [Vu], formula (1.9), and [VV]. Next, as is shown in [VV], the function $\eta_{K, 2}(t)-t$ is strictly increasing in $t$ if $K>1$. This and the proof of 2.13 give the following result: 
2.16. Theorem. A K-QC map $f: R^{2} \rightarrow R^{2}$ is $s-\mathrm{QS}$, where $s=s(K)$ is the unique solution of the transcendental equation

$$
\eta_{K, 2}(1 / s)=s+1 / s
$$

2.17. Remark. One can show by Theorem 2.16 that for $K$ sufficiently close to $1, f$ is $s$-QS with $s=\sqrt[4]{K-1}$.

\section{Simplexes}

In this section we present auxiliary material on the volume and the flatness of a simplex. We also consider the distortion of a simplex under an $s$-QS map of its vertices.

3.1. Volume of a simplex. Let $\bar{a}=\left(a_{0}, \ldots, a_{k}\right)$ be a sequence of $k+1$ points in $E$. We set $d_{i j}=\left|a_{i}-a_{j}\right|$. The Cayley-Menger determinant of $\bar{a}$ is defined as

$$
\Gamma(\bar{a})=\left|\begin{array}{ccccc}
0 & 1 & 1 & \cdots & 1 \\
1 & 0 & d_{01}^{2} & \cdots & d_{0 k}^{2} \\
1 & d_{10}^{2} & 0 & \cdots & d_{1 k}^{2} \\
\vdots & \vdots & \vdots & & \vdots \\
1 & d_{k 0}^{2} & d_{k 1}^{2} & \cdots & 0
\end{array}\right|
$$

This determinant is nonzero if and only if $\bar{a}$ is affinely independent. In this case, the volume of the $k$-simplex $\Delta=a_{0} \ldots a_{k}$ is

$$
m_{k}(\Delta)=\frac{1}{2^{k / 2} k !} \sqrt{|\Gamma(\bar{a})|} .
$$

Moreover, $\Gamma(\bar{a})>0$ if and only if $k$ is odd. For these results, see [Be], p. 238 or [Bl], p. 98.

We let $\alpha(k)$ denote the volume of a unit $k$-simplex, that is, a regular simplex of edge 1. Explicitly,

$$
\alpha(k)=\frac{\sqrt{k+1}}{2^{k / 2} k !} ;
$$

see [So], p. 125. 


\subsection{Lemma. For every $k$-simplex $\Delta$ we have $m_{k}(\Delta) \leq \alpha(k) d(\Delta)^{\mathrm{k}}$.}

Proof. We may assume that $d(\Delta)=1$. The lemma is clearly true for $k=1$. Proceeding inductively, assume that it is true for all $(k-1)$-simplexes. Then the $(k-1)$-measure of each face of $\Delta$ is at most $\alpha(k-1)$. Thus $m_{k-1}(\partial \Delta) \leq$ $m_{k-1}\left(\partial \Delta_{0}\right)$ where $\Delta_{0}$ is a unit $k$-simplex. This implies that $m_{k}(\Delta) \leq m_{k}\left(\Delta_{0}\right)=$ $\alpha(k)$ by the isoperimetric property of the unit simplex [Ha], (187), p. 273.

3.6. Flatness of a simplex. We define two numbers $\rho(\Delta)$ and $\sigma(\Delta)$ to describe the flatness of a $k$-simplex $\Delta=a_{0} \ldots a_{k}$. First we set

$$
\sigma(\Delta)=\frac{d(\Delta)^{k}}{m_{k}(\Delta)}
$$

Observe that the diameter $d(\Delta)$ is the length of the longest edge of $\Delta$. To define $\rho(\Delta)$ we let $b_{j}(\Delta)$ denote the distance between the vertex $a_{j}$ and the $(k-1)$ plane spanned by the opposite $(k-1)$-face of $\Delta$. Then we set

$$
b(\Delta)=\min \left\{b_{j}(\Delta): 0 \leq j \leq k\right\}, \quad \rho(\Delta)=\frac{d(\Delta)}{b(\Delta)} .
$$

The number $\rho(\Delta)$ has been used in [Vä3].

The numbers $\rho(\Delta)$ and $\sigma(\Delta)$ are obviously invariant under similarity maps. They are large if $\Delta$ is very flat. On the other hand, they cannot be arbitrarily small. For example, if follows from 3.5 that $\sigma(\Delta)$ is minimized by the regular $k$-simplex. Thus $\sigma(\Delta) \geq 1 / \alpha(k)$ for every $k$-simplex. Clearly we have always $\rho(\Delta) \geq 1$.

For a 2 -simplex $\Delta$ we obviously have $2 m_{2}(\Delta)=b(\Delta) d(\Delta)$ and hence

$$
\sigma(\Delta)=2 \rho(\Delta)
$$

We next prove the inequalities

$$
2^{(k-1) / 2}(k-1) ! \sqrt{k} \rho(\Delta) \leq \sigma(\Delta) \leq k ! \rho(\Delta)^{k(k+1) / 2}
$$

for every $k$-simplex $\Delta$.

We may assume that $\Delta=a_{0} \ldots a_{k}$ with $b(\Delta)=b_{k}(\Delta)$. Let $\Delta_{1}$ be the face $a_{0} \ldots a_{k-1}$ of $\Delta$. Then

$$
m_{k}(\Delta)=b(\Delta) m_{k-1}\left(\Delta_{1}\right) / k
$$

By 3.5 we have 


$$
m_{k-1}\left(\Delta_{1}\right) \leq \alpha(k-1) d\left(\Delta_{1}\right)^{k-1} \leq \alpha(k-1) d(\Delta)^{k-1} .
$$

Recalling (3.4) we obtain the first inequality of (3.10). It is sharp, since it holds as an equality for a regular $k$-simplex.

The second inequality of (3.10) holds for $k=1$ in the form $\sigma(\Delta)=1=$ $\rho(\Delta)$. Proceeding inductively, assume that it is true for $(k-1)$-simplexes. We have

$$
\sigma(\Delta)=\frac{k d(\Delta)^{k}}{b_{k}(\Delta) m_{k-1}(\Delta)}, \quad b(\Delta) \leq b\left(\Delta_{1}\right) \leq d\left(\Delta_{1}\right)
$$

and hence

$$
\sigma(\Delta) \leq k \frac{d(\Delta)^{k}}{b(\Delta)^{k}} \frac{d\left(\Delta_{1}\right)^{k-1}}{m_{k-1}\left(\Delta_{1}\right)}=k \rho(\Delta)^{k} \sigma\left(\Delta_{1}\right) .
$$

Since $\rho\left(\Delta_{1}\right) \leq \rho(\Delta)$, this and the inductive hypothesis yield the second inequality of (3.10), which is not sharp.

3.11. QS maps of simplexes. Suppose that $\Delta=a_{0} \ldots a_{k}$ is a $k$-simplex in $E$. We let $\Delta^{0}=\left\{a_{0}, \ldots, a_{k}\right\}$ denote the set of vertices of $\Delta$. Let $E^{\prime}$ be another inner product space and let $f: \Delta^{0} \rightarrow E^{\prime}$ be an $s$-QS map. If $f$ is a similarity, it preserves the flatness numbers $\rho(\Delta)$ and $\sigma(\Delta)$. It is therefore natural to guess that for small $s, f \Delta^{0}$ defines a simplex whose flatness differs only slightly from the flatness of $\Delta$. This is easy to prove by normal families, but we want to get explicit estimates. We prove only what is actually needed in Section 4.

3.12. Lemma. Let $r \geq 1$ and let $k$ be a positive integer. Then there is a number $s^{*}=s^{*}(r, k)>0$ and a positive decreasing function $s \mapsto \lambda(s, r, k)$, defined on $\left[0, s^{*}\right]$, such that $\lambda(s, r, k) \rightarrow 1$ as $s \rightarrow 0$ and such that the following statement is true:

Let $\Delta=a_{0} \ldots a_{k} \subset E$ be a $k$-simplex with $\rho(\Delta) \leq r$ and $d(\Delta)=\left|a_{0}-a_{1}\right|$ $=1$. Let $f: \Delta^{0} \rightarrow E^{\prime}$ be an $s-\mathrm{QS}$ map with $\left|f a_{0}-f a_{1}\right|=1$. If $s \leq 1 /(3 r)$, then

$$
(1-3 r s)\left|a_{i}-a_{j}\right| \leq\left|f a_{i}-f a_{j}\right| \leq(1+3 r s)\left|a_{i}-a_{j}\right|
$$

for all $i, j$. If $s \leq s^{*}(r, k)$, then

$$
|\Gamma(f \bar{a})| \geq \lambda(s, r, k)|\Gamma(\bar{a})|,
$$

where $\Gamma$ is the Cayley-Menger determinant (3.2) and $f \bar{a}=\left(f a_{0}, \ldots, f a_{k}\right)$.

The bounds $s^{*}(r, k)$ and $\lambda(s, r, k)$ are explicit and are explained in 3.16 and, 
for $k=2$, in 3.19 .

Proof. Suppose that $\Delta=a_{0} \ldots a_{k}$ and $\rho(\Delta) \leq r$. Write $s_{1}=1 /(3 r)$ and suppose that $f: \Delta^{0} \rightarrow E^{\prime}$ is $s$-QS with $0<s \leq s_{1}$. Set

$$
d_{i \jmath}=\left|a_{\imath}-a_{j}\right|, d=\min _{i \neq j} d_{i j}, d_{i j}^{\prime}=\left|f a_{i}-f a_{j}\right| .
$$

Since $d_{i j} \geq b_{j}(\Delta) \geq b(\Delta)=1 / \rho(\Delta) \geq 1 / r$, we have

$$
d \geq 1 / r .
$$

To prove (1) we may assume that $i \neq 0$ and $i \neq j$. Since $d \leq d_{k l} \leq d_{01}=1$ for all $k \neq l$, we have

$$
\frac{d_{i j}}{d_{0 \imath}} \leq \frac{1}{d} \leq r=\frac{1}{3 s_{1}}<\frac{1}{s}
$$

and $d_{0 i} / d_{01} \leq 1<1 / s$. Hence the $s$-quasisymmetry of $f$ implies

$$
d_{i j}^{\prime}=\frac{d_{i j}^{\prime}}{d_{0 i}^{\prime}} \frac{d_{0 i}^{\prime}}{d_{01}^{\prime}} \leq\left(\frac{d_{i j}}{d_{0 i}}+s\right)\left(\frac{d_{0 i}}{d_{01}}+s\right)=d_{i j}(1+u),
$$

where (3.13) yields

$$
u=s / d_{0 i}+s d_{0 i} / d_{\imath j}+s^{2} / d_{i j} \leq s / d+s / d+s^{2} / d \leq 3 s / d \leq 3 r s,
$$

and we obtain the second inequality of (1).

Similar arguments give

$$
\frac{1}{d_{i j}^{\prime}}=\frac{d_{0 i}^{\prime}}{d_{\imath j}^{\prime}} \frac{d_{01}^{\prime}}{d_{0 i}^{\prime}} \leq\left(\frac{d_{0 i}}{d_{i j}}+s\right)\left(\frac{d_{01}}{d_{0 i}}+s\right)=\frac{1}{d_{i j}(1-v)},
$$

where

$$
v=\frac{s d_{0 i}^{2}+s d_{i j}+s^{2} d_{i j} d_{0 i}}{\left(d_{0 i}+s d_{i j}\right)\left(1+s d_{0 i}\right)}<\frac{s+s+s^{2}}{d} \leq 3 r s \leq 1
$$

This gives the first inequality of (1).

We turn to the determinant $\Gamma(\bar{a})$. We can write

$$
|\Gamma(\bar{a})|=p-q, \quad p=\sum_{\mu} p_{\mu}, \quad q=\sum_{\nu} q_{\nu}
$$

where each $p_{\mu}$ and $q_{\nu}$ is a product of $k$ numbers of the form $d_{i j}^{2}$. The number of terms of each sum is less than $(k+2) ! / 2$. Replacing $d_{i j}$ by $d_{i j}^{\prime}$ we similarly obtain the numbers $p_{\mu}^{\prime}, q_{\nu}^{\prime}, p^{\prime}, q^{\prime}$ with 


$$
|\Gamma(f \bar{a})|=p^{\prime}-q^{\prime}
$$

Writing $t=3 r s$ we obtain from (1):

and hence

$$
(1-t)^{2 k} p_{\mu} \leq p_{\mu}^{\prime} \leq(1+t)^{2 k} p_{\mu}
$$

$$
(1-t)^{2 k} p \leq p^{\prime} \leq(1+t)^{2 k} p .
$$

Similar relations hold for $q$ and $q^{\prime}$. Thus

$$
|\Gamma(f \bar{a})|=p^{\prime}-q^{\prime} \geq(1-t)^{2 k} p-(1+t)^{2 k} q=\lambda_{0}|\Gamma(\bar{a})|,
$$

where

$$
\lambda_{0}=(1-t)^{2 k}-\frac{q}{p-q}\left[(1+t)^{2 k}-(1-t)^{2 k}\right] .
$$

Since $d_{\imath j} \leq 1$, we have $q<(k+2) ! / 2$. Next, because $d(\Delta)=1$, we obtain from (3.3) and (3.10) that

$$
p-q=|\Gamma(\bar{a})|=2^{k}(k !)^{2} m_{k}(\Delta)^{2}=\frac{2^{k}(k !)^{2}}{\sigma(\Delta)^{2}} \geq 2^{k} r^{-k(k+1)} .
$$

Consequently, we have $|\Gamma(f \bar{a})| \geq \lambda|\Gamma(\bar{a})|$ with

$$
\lambda=\lambda(s, r, k)=(1-t)^{2 k}-\frac{(k+2) ! r^{k(k+1)}}{2^{k+1}}\left[(1+t)^{2 k}-(1-t)^{2 k}\right],
$$

where $t=3 r s$. Clearly $\lambda(s, r, k)$ is decreasing in $s$ and $\lambda(s, r, k) \rightarrow 1$ as $s \rightarrow 0$. Choose $s^{*}=s^{*}(r, k)$ such that $s^{*} \leq s_{1}=1 /(3 r)$ and such that $\lambda(s, r, k)>0$ for $s \in\left[0, s^{*}\right]$. Then the lemma is true with these $s^{*}$ and $\lambda$.

3.16. Bounds in 3.12. The numbers $s^{*}(r, k)$ and $\lambda(s, r, k)$ obtained in the proof of 3.12 are explicitly computable but rather complicated. We shall next give simpler but somewhat less accurate bounds. The function $g(x)=(1+x)^{2 k}$ is convex and hence above its tangent:

$$
g(x) \geq 1+2 k x
$$

for every real $x$. Moreover, $0 \leq g^{\prime}(x)=2 k(1+x)^{2 k-1} \leq 2^{2 k} k$ for $|x| \leq 1$, and hence

$$
0 \leq g(x)-g(-x) \leq 2^{2 k+1} k x
$$

for $0 \leq x \leq 1$. Since $t=3 r s \leq 1$ in (3.15), we obtain 


$$
\begin{aligned}
\lambda(s, r, k) & \geq 1-2 k t-2^{k}(k+2) ! k r^{k(k+1)} t \\
& =1-a(r, k) s,
\end{aligned}
$$

where

$$
a(r, k)=6 k r\left(1+2^{k-1}(k+2) ! r^{k(k+1)}\right) .
$$

Hence Lemma 3.12 is valid with

$$
s^{*}(r, k)=1 /(2 a(r, k)), \lambda(s, r, k)=1-a(r, k) s .
$$

In the case $k=2$ better estimates are given in 3.19 .

3.19. The case $k=2$. We give an independent treatment of the case $k=2$ of Lemma 3.12. Indeed, we show that one can choose

$$
s^{*}(r, 2)=1 /\left(8 r^{2}\right), \lambda(s, r, 2)=1-7 r^{2} s .
$$

Assume that $\Delta=a_{0} a_{1} a_{2}$ and $f: \Delta^{0} \rightarrow E^{\prime}$ are as in 3.12. For $b=d\left(a_{2}\right.$, $a_{0} a_{1}$ ) we have

$$
1 / b=\rho(\Delta) \leq r, \quad m(\Delta)=b / 2, \quad b \leq \sqrt{3} / 2, \quad r \geq 2 / \sqrt{3} .
$$

For $j=0,1$ write

$$
u_{j}=\left|a_{j}-a_{2}\right|, \quad v_{j}=\left|f a_{j}-f a_{2}\right|, \quad 2 p=1+u_{0}+u_{1}, \quad 2 q=1+v_{0}+v_{1} .
$$

We may assume that $u_{0} \leq u_{1}$. Let $\Delta^{\prime}$ be the 2 -simplex with vertices $f a_{0}, f a_{1}, f a_{2}$. The classical Heron's formula gives

$$
\frac{m\left(\Delta^{\prime}\right)^{2}}{m(\Delta)^{2}}=\frac{q(q-1)\left(q-v_{0}\right)\left(q-v_{1}\right)}{p(p-1)\left(p-u_{0}\right)\left(p-u_{1}\right)} .
$$

Assume that $s \leq 1 /\left(8 r^{2}\right)$. Then (3.21) implies that

$$
s \leq b \sqrt{3} / 16<b, \quad s \leq 3 / 32<1 / 10 .
$$

Hence $1 / u_{j}<1 / b<1 / s$ and $u_{j} \leq 1<1 / s$. Consequently, the $s$-quasisymmetry of $f$ gives

$$
\frac{1}{v_{j}} \leq \frac{1}{u_{j}}+s \leq \frac{1+s}{u_{j}}, \quad v_{j} \leq u_{j}+s
$$

which implies

$$
q \geq p /(1+s)
$$

We next show that 


$$
p-1 \geq 2 /\left(3 r^{2}\right) .
$$

For given $b$, the sum $u_{0}+u_{1}$ is minimal when $u_{0}=u_{1}$. Thus $u_{0}+u_{1} \geq(1+$ $\left.4 b^{2}\right)^{1 / 2}$. Since (3.21) implies that $4 b^{2} \leq 3$, this and (3.21) yield

$$
u_{0}+u_{1} \geq 1+4 b^{2} / 3 \geq 1+4 /\left(3 r^{2}\right) \text {, }
$$

and we obtain (3.24)

The inequalities (3.22) and (3.24) imply

$$
\frac{q-1}{p-1} \geq \frac{1}{1+s}\left(1-\frac{s}{2(p-1)}\right) \geq \frac{1-3 r^{2} s / 4}{1+s} .
$$

To estimate the numbers $q-v_{j}$ we write $k=1-j$. Since $1+u_{k}=2 p-u_{j}$, (3.22) gives

$$
2\left(q-v_{j}\right)=1+v_{k}-v_{j} \geq \frac{2\left(p-u_{j}\right)-s\left(u_{j}+s\right)}{1+s},
$$

and hence

$$
\frac{q-v_{j}}{p-u_{j}} \geq \frac{1-c_{j} s}{1+s}, \quad c_{j}=\frac{u_{j}+s}{2\left(p-u_{j}\right)}
$$

For $j=0$ we have $2\left(p-u_{0}\right)=1+u_{1}-u_{0} \geq 1$, and hence $c_{0} \leq 1+s$. Since $s \leq 1 / 10$, we obtain

$$
\frac{q-v_{0}}{p-u_{0}} \geq \frac{1-11 s / 10}{1+s}
$$

For $j=1$ we observe that $2 p \leq 3,2(p-1) \leq 1,2\left(p-u_{0}\right) \leq 2, m(\Delta) \geq$ $1 /(2 r)$, and hence Heron's formula gives $p-u_{1} \geq 1 /\left(3 r^{2}\right)$. Thus

$$
c_{1} \leq 3 r^{2}(1+1 / 10) / 2=33 r^{2} / 20 \text {, }
$$

and hence

$$
\frac{q-v_{1}}{p-u_{1}} \geq \frac{1-33 r^{2} s / 20}{1+s} .
$$

Joining the estimates (3.23), (3.25), (3.26) and (3.27) yields

$$
\frac{m\left(\Delta^{\prime}\right)^{2}}{m(\Delta)^{2}} \geq \frac{1}{(1+s)^{4}}\left(1-\frac{3 r^{2} s}{4}\right)\left(1-\frac{11 s}{10}\right)\left(1-\frac{33 r^{2} s}{20}\right) \text {. }
$$

Since $(1+s)^{-4} \geq 1-4 s$ and since 


$$
\left(1-t_{1}\right) \cdots\left(1-t_{n}\right) \geq 1-\left(t_{1}+\cdots+t_{n}\right)
$$

for all positive $t_{j}$, these inequalities and (3.21) easily give $m\left(\Delta^{\prime}\right)^{2} \geq \lambda m(\Delta)^{2}$ with $\lambda$ as in 3.20 .

If we replace the condition $s \leq 1 /\left(8 r^{2}\right)$ by $s \leq 2 /\left(5 r^{2}\right)$, it is easy to show by the same arguments that we still have $m\left(\Delta^{\prime}\right)>0$. Since $\sigma(\Delta)=2 \rho(\Delta)$ by (3.9), we obtain the following result, which is useful in Section 5:

3.29. Lemma. Suppose that $\Delta$ is a 2-simplex in $E$ and that $f: \Delta^{0} \rightarrow E^{\prime}$ is an $s$-QS map with $s \leq 3 /\left(2 \sigma(\Delta)^{2}\right)$. Then $f \Delta^{0}$ is not contained in a line.

\section{Thickness and quasisymmetry}

4.1. Thickness. This section gives the main result of the paper: The thickness of a set can change only slightly under an $s$-QS map with small $s$.

As before, $E$ and $E^{\prime}$ will denote inner product spaces. Let $q>0$ and let $k$ be a positive integer. We recall from the introduction that a set $A \subset E$ is $(q, k)$-thick if for each $x \in A$ and $r>0$ such that $A \backslash \bar{B}(x, r) \neq \emptyset$ there is a $k$-simplex $\Delta$ with vertices in $A \cap \bar{B}(x, r)$ such that $m_{k}(\Delta) \geq q r^{k}$. The volume of a $k$-simplex $\Delta$ in $\bar{B}(x, r)$ is maximal when $\Delta$ is a regular $k$-simplex with edge $(2 k+2)^{1 / 2} k^{-1 / 2} r$. From this and from (3.4) it follows that the number $q$ of a $(q, k)$-thick set satisfies the condition

$$
0<q \leq q_{0}(k)=\frac{(k+1)^{(k+1) / 2}}{k^{k / 2} k !} .
$$

4.3. THEOREM. Let $k$ be a positive integer and let $q>0$. There is a number $s_{0}=s_{0}(q, k)>0$ and a positive decreasing function $s \mapsto q_{1}(q, s, k)$, defined on $\left[0, s_{0}\right]$, such that:

(1) $\lim _{s \rightarrow 0} q_{1}(q, s, k)=q$.

(2) If $A \subset E$ is $(q, k)$-thick and if $f: A \rightarrow E^{\prime}$ is $s-Q S, s \leq s_{0}$, then $f A$ is $\left(q_{1}, k\right)$-thick.

The bounds are explicit.

Proof. Let $A \subset E$ and $f: A \rightarrow E^{\prime}$ be as in (2). Suppose that $x_{0} \in A, y_{0}=f x_{0}$, and that $r>0$ such that the set $F^{\prime}=f A \backslash \bar{B}\left(y_{0}, r\right)$ is not empty. We must find a $k$-simplex $\Delta^{\prime}$ with vertices in $\bar{B}\left(y_{0}, r\right) \cap f A$ and an estimate

$$
m_{k}\left(\Delta^{\prime}\right) \geq q_{1}(q, s, k) r^{k},
$$


valid on an interval $0 \leq s \leq s_{0}(q, k)$. We may assume that $s>0$, since in the case $s=0, f$ is a similarity, and we can choose $q_{1}=q$.

We set

$$
F=f^{-1} F^{\prime}, r_{1}=d\left(F, x_{0}\right), r_{2}=r_{1} /(1+s)
$$

Then

$$
\emptyset \neq F \subset A \backslash B\left(x_{0}, r_{1}\right) .
$$

We assume that $s \leq 1 / 2$ and give more restrictions on $s$ later.

By the definition of $(q, k)$-thickness, there is a $k$-simplex $\Delta=a_{0} \ldots a_{k}$ with vertices in $A \cap \bar{B}\left(x_{0}, r_{2}\right)$ such that

$$
m_{k}(\Delta) \geq q r_{2}^{k} .
$$

We choose the order of the vertices so that

$$
\left|a_{0}-a_{1}\right|=d(\Delta), \quad\left|a_{0}-x_{0}\right| \geq d(\Delta) / 2 .
$$

Write $a_{j}^{\prime}=f a_{j}$ and $\Delta^{\prime}=a_{0}^{\prime} \ldots a_{k}^{\prime}$. Since $\left|a_{j}-x_{0}\right| \leq r_{2}<r_{1}$, the points $a_{j}^{\prime}$ are in $\bar{B}\left(y_{0}, r\right) \cap f A$. Hence it suffices to show that $\Delta^{\prime}$ satisfies (4.4) with suitable numbers $q_{1}$ and $s_{0}$.

We can use auxiliary similarities to normalize the situation so that

$$
d(\Delta)=\left|a_{0}-a_{1}\right|=1, \quad\left|a_{0}^{\prime}-a_{1}^{\prime}\right|=1 .
$$

Choose $x_{1} \in F$ such that

$$
\left|x_{1}-x_{0}\right| \leq(1+s) r_{1} \text {. }
$$

We want to apply the $s$-quasisymmetry of $f$ to the triples $\left(x_{0}, x_{1}, a_{0}\right)$ and $\left(a_{0}, x_{0}\right.$, $\left.a_{1}\right)$. For this we need the inequalities

$$
\frac{\left|x_{1}-x_{0}\right|}{\left|a_{0}-x_{0}\right|} \leq \frac{1}{s}, \quad \mid \frac{x_{0}-a_{0} \mid}{\left|a_{1}-a_{0}\right|} \leq \frac{1}{s} .
$$

Setting

$$
s_{1}(q, k)=\frac{1}{5}\left(\frac{q}{\alpha(k)}\right)^{1 / k}
$$

where $\alpha(k)$ is as in (3.4), we show that (4.6) is true if $s \leq s_{1}(q, k)$ and $s \leq 1 / 2$. Indeed, we have

$$
\frac{\left|x_{1}-x_{0}\right|}{\left|a_{0}-x_{0}\right|} \leq \frac{(1+s) r_{1}}{d(\Delta) / 2} \leq 3 r_{1}, \quad \frac{\left|x_{0}-a_{0}\right|}{\left|a_{1}-a_{0}\right|}=\left|x_{0}-a_{0}\right|<r_{1} .
$$


From Lemma 3.5 and from (4.5) we obtain

$$
3 r_{1}=3(1+s) r_{2}<5 r_{2} \leq 5\left(m_{k}(\Delta) / q\right)^{1 / k} \leq 1 / s_{1}(q, k),
$$

and (4.6) follows.

From now on, we assume that $s \leq s_{1}(q, k)$ and $s \leq 1 / 2$. Since $f x_{1} \in F^{\prime}$ and since $f$ is $s-Q S,(4.6)$ implies

$$
\begin{aligned}
\frac{r}{d\left(\Delta^{\prime}\right)} & \leq \frac{\left|f x_{1}-y_{0}\right|}{\left|a_{0}^{\prime}-y_{0}\right|} \mid \frac{y_{0}-a_{0}^{\prime} \mid}{\left|a_{1}^{\prime}-a_{0}^{\prime}\right|} \leq\left(\frac{\left|x_{1}-x_{0}\right|}{\left|a_{0}-x_{0}\right|}+s\right)\left(\frac{\left|x_{0}-a_{0}\right|}{\left|a_{1}-a_{0}\right|}+s\right) \\
& \leq\left|x_{1}-x_{0}\right|+s\left(\left|x_{0}-a_{0}\right|+\frac{\left|x_{1}-x_{0}\right|}{\left|a_{0}-x_{0}\right|}+s\right) \\
& \leq(1+s) r_{1}+s\left(r_{2}+2(1+s) r_{1}+s\right) .
\end{aligned}
$$

Since $s \leq 1 / 2$ and

$$
1=d(\Delta) \leq 2 r_{2}
$$

this yields

$$
r / d\left(\Delta^{\prime}\right) \leq(1+9 s) r_{2}
$$

By (3.10) we have

$$
\rho(\Delta) \leq c_{k}^{\prime} \sigma(\Delta)=c_{k}^{\prime} / m_{k}(\Delta)
$$

where

$$
1 / c_{k}^{\prime}=2^{(k-1) / 2}(k-1) ! \sqrt{k} .
$$

Since (4.5) and (4.8) give $m_{k}(\Delta) \geq q / 2^{k}$, we obtain $\rho(\Delta) \leq c_{k} / q$ where $c_{k}=2^{k} c_{k}^{\prime}$. Write $s_{2}(q, k)=q /\left(3 c_{k}\right)$ and assume from now on that $s \leq s_{2}(q, k)$. Since $s \leq$ $1 /(3 \rho(\Delta)), 3.12(1)$ gives

$$
d\left(\Delta^{\prime}\right) \leq(1+3 \rho(\Delta) s) d(\Delta)=1+3 \rho(\Delta) s .
$$

By (4.9) this implies

$$
r \leq(1+9 s)\left(1+3 c_{k} s / q\right) r_{2} .
$$

Since $\rho(\Delta) \geq 1$ for every simplex $\Delta$, we have $c_{k} / q \geq 1$. Let $s^{*}(r, k)$ and $\lambda(s, r, k)$ be the functions given by 3.12 . We set

$$
s_{3}(q, k)=s^{*}\left(c_{k} / q, k\right), \quad \lambda_{1}(q, s, k)=\lambda\left(s, c_{k} / q, k\right)
$$

and assume that $s \leq s_{3}(q, k)$. Now (3.3), $3.12(2),(4.5)$ and (4.10) give (4.4) with 


$$
q_{1}(q, s, k)=\frac{q \lambda_{1}(q, s, k)^{1 / 2}}{(1+9 s)^{k}\left(1+3 c_{k} s / q\right)^{k}} .
$$

This is valid for

$$
s \leq s_{0}(q, k)=\min \left\{1 / 2, s_{1}(q, k), s_{2}(q, k), s_{3}(q, k)\right\} .
$$

Since $q_{1}(q, s, k) \rightarrow q$ as $s \rightarrow 0$, the theorem is proved.

4.13. Bounds in 4.3. The bounds $s_{0}(q, k)$ and $q_{1}(q, s, k)$ obtained in the proof of Theorem 4.3 are explicitly computable but somewhat complicated. We shall give more explicit bounds in the case $k=2$.

The function $s_{0}(q, 2)$ was obtained as the minimum of the numbers $1 / 2$, $s_{1}(q, 2), s_{2}(q, 2)$ and $s_{3}(q, 2)$. With the notation of the proof we have $c_{2}=2$. By (3.20) and (4.10) we can choose

$$
s_{3}(q, 2)=s^{*}(2 / q, 2)=q^{2} / 32 .
$$

Since $q \leq q_{0}(2)=3 \sqrt{3} / 4$ by $(4.2)$, it is easy to see that $s_{0}(q, 2)=q^{2} / 32$.

From (3.20) and (4.11) we obtain

$$
\lambda_{1}(q, s, 2)=1-28 s / q^{2} .
$$

By (4.12) this gives the bound

$$
q_{1}(q, s, 2)=q\left(1-\frac{28 s}{q^{2}}\right)^{1 / 2}(1+9 s)^{-2}\left(1+\frac{6 s}{q}\right)^{-2} .
$$

This can be simplified by the elementary inequalities $\sqrt{1-t} \geq 1-t,(1+t)^{-1}$ $>1-t$, (3.28), and the upper bound $q \leq 3 \sqrt{3} / 4$; see (4.2). We obtain the lower bound

$$
q_{1}(q, s, 2)>q\left(1-\frac{74 s}{q^{2}}\right)
$$

which is positive for $s<q^{2} / 74$. Hence we can choose $s_{0}(q, 2)=q^{2} / 75$ and $q_{1}(q, s, 2)=q\left(1-74 s / q^{2}\right)$.

4.14. Theorem. Let $k$ be a positive integer and let $q>0$. Then there is $K_{0}=$ $K_{0}(q, k)>1$ and an increasing function $K \mapsto q_{2}(q, K, k)$, defined on $\left[1, K_{0}\right]$, such that

(1) $\lim _{K \rightarrow 1} q_{2}(q, K, k)=q$.

(2) If $A \subset R^{n}$ is $(q, k)$-thick and if $f: R^{n} \rightarrow R^{n}$ is $K-\mathrm{QC}$ with $K \leq K_{0}$, then 
$f A$ is $\left(q_{2}, k\right)$-thick.

The bounds are explicit.

Proof. This follows directly from Theorems 2.13 and 4.3 .

4.15. Remark. The intuitive meaning of the $(q, k)$-thickness is that the sets with this property avoid in a uniform way all $k$-dimensional affine subspaces. Sets with the opposite property, that is, sets which can be well approximated by $k$-dimensional planes were studied in [MV].

\section{Ares}

5.1. QS and QC arcs. By an arc in a topological space we mean a set $\alpha$ homeomorphic to $[0,1]$. If there is an $s$-QS homeomorphism $f: \alpha \rightarrow[0,1]$, we say that $\alpha$ is an $s$-quasisymmetric arc. Let $\dot{R}^{2}=R^{2} \cup\{\infty\}$ be the one-point extension of $R^{2}$. If $\alpha \subset \dot{R}^{2}$ and there is a $K-Q C$ map $f: \dot{R}^{2} \rightarrow \dot{R}^{2}$ carrying $\alpha$ onto a line segment, $\alpha$ is a $K$-quasiconformal arc or briefly a $K$-quasiarc.

An $s$-QS arc $\alpha \subset R^{2}$ is always a $K$-quasiarc with $K=K(s) \rightarrow 1$ as $s \rightarrow 0$. Moreover, the $K$-QC map $f$ can be chosen to fix the point $\infty$. We prove this in 5.2 , but the result is well known. Conversely, if $f: \dot{R}^{2} \rightarrow \dot{R}^{2}$ is $K-\mathrm{QC}$ and if $f(\infty)=\infty$, then $f$ maps each line segment onto an $s-Q S$ arc with $s=s(K) \rightarrow 0$ as $K \rightarrow 1$. This follows from 2.13. However, if $f(\infty) \neq \infty$, even a conformal mapping $f$ can map a line segment onto a circular arc of angle close to $2 \pi$, and then $\alpha$ is not an $s-\mathrm{QS}$ arc with small $s$.

In this section we derive upper bounds for the thickness of $s-\mathrm{QS}$ and $K-\mathrm{QC}$ arcs. By means of an example we also study the sharpness of these bounds. We formulate the results for 2 -thickness, but bounds for the $k$-thickness of $s$-QS arcs can be obtained by similar methods.

Every arc is trivially 1 -thick. For $k \geq 2$, a $k$-thick arc cannot have a tangent at any point. In particular, a rectifiable arc in $R^{n}$ is never $k$-thick for $k \geq 2$.

5.2. Lemma. Suppose that $\alpha$ is an $s-\mathrm{QS}$ arc in $R^{2}$. Then there is a K-QC map $f: R^{2} \rightarrow R^{2}$ such that $f \alpha$ is a line segment and such that $K=K(s) \rightarrow 1$ as $s \rightarrow 0$.

Proof. Choose an $s$-QS homeomorphism $g: \alpha \rightarrow[0,1]$. Then $g$ is weakly $(1+s)-\mathrm{QS}$ in the sense of $\left[\mathrm{TV}_{1}\right]$. By [Vä4], Th. $2.9, g$ is $\eta$-QS with $\eta=\eta_{s}$. Hence $g^{-1}$ is $\eta^{\prime}$-QS with $\eta^{\prime}(t)=\eta^{-1}\left(t^{-1}\right)^{-1}$. Thus $g$ is $\theta$-quasimöbius with $\theta=$ $\theta_{s}$, by [Vä], Th. 3.2. It follows that the distortion of $\alpha$ in the sense of Rickman [Ri] 
is at most $2 \theta(1)$. By Theorem 1 of [Ri] and by the subsequent Remark, there is $K_{1}=K_{1}(s)$ and a $K_{1}-\mathrm{QC}$ map $f_{1}: \dot{R}^{2} \rightarrow \dot{R}^{2}$ carrying $\alpha$ onto the positive half $R_{+}^{1}$ of the real axis. The map $f_{1}$ is $\theta^{\prime}$-quasimöbius with $\theta^{\prime}=\theta_{s}^{\prime}$; cf. [Vä2], p. 232.

Write $y=f(\infty)$ and choose $x \in \alpha$ with $|f x|=y$. Let $a$ and $b$ be the endpoints of $\alpha$ with $f a=0, f b=\infty$. Considering the quadruple $(x, b, a, \infty)$ we obtain

$$
\frac{|f x|}{|f x-y|} \leq \theta^{\prime}\left(\frac{|a-x|}{|a-b|}\right) \leq \theta^{\prime}(\eta(1)) .
$$

It follows that $|\arg y|$ is bounded from below by a positive constant depending only on $s$. Hence there is $K_{2}=K_{2}(s)$ and a $K_{2}-\mathrm{QC}$ map $f_{2}: \dot{R}^{2} \rightarrow \dot{R}^{2}$ such that $f_{2} \mid R_{+}^{1}=$ id and $f_{2} y$ lies in the negative half of the real axis. Let $f_{3}$ be a Möbius map which carries $\left(f_{2} y, 0, \infty\right)$ onto $(\infty, 0,1)$. Then the composite map $f=f_{3} f_{2} f_{1}$ is $K_{1} K_{2}-\mathrm{QC}$ with $f(\infty)=\infty$ and $f \alpha=[0,1]$.

The construction above is valid for every $s>0$. For small $s$ we can make use of the fact that the line segment $[0,1]$ has the QS extension property (QSEP) in $R^{2}$; see [Vä3], Th. 4.6. The map $g^{-1}:[0,1] \rightarrow \alpha$ is $s_{1}-\mathrm{QS}$, where $s_{1}=s_{1}(s) \rightarrow 0$ as $s \rightarrow 0$; cf. [Pa], Lemma 1.4. By the QSEP of $[0,1]$, there is a universal constant $s_{0}>0$ such that if $0 \leq s \leq s_{0}$, we can extend $g^{-1}$ to an $s_{2}-\mathrm{QS}$ map $F: R^{2} \rightarrow R^{2}$, where $s_{2}=s_{2}(s) \rightarrow 0$ as $s \rightarrow 0$. Then $f=F^{-1}$ is the desired map by 2.5 .

5.3. Lemma. Suppose that $\alpha$ is an $s-\mathrm{QS}$ arc in $E$ and that $\alpha$ contains the vertices of a 2 -simplex $\Delta$. Then

$$
\sigma(\Delta)^{2} \geq \frac{3}{2 s}
$$

Proof. This follows directly from 3.29.

5.4. Remark. Lemma 5.3 is nontrivial only for $s<9 / 32$, since we always have $\sigma(\Delta) \geq 1 / \alpha(2)=4 / \sqrt{3}$.

5.5. THEOREM. Suppose that $\alpha$ is an s-QS arc in an inner product space $E$ and that $\alpha$ is $(q, 2)$-thick. Then $q \leq \sqrt{11 s}$.

Proof. Assume that $\alpha$ is $(q, 2)$-thick. Choose $x \in \alpha$ and $r>0$ such that $\alpha \backslash \bar{B}(x, r) \neq \emptyset$. Then there is a 2 -simplex $\Delta$ with vertices in $\alpha \cap \bar{B}(x, r)$ such that $m_{2}(\Delta) \geq q r^{2}$. Since $d(\Delta) \leq 2 r$, we have $\sigma(\Delta) \leq 4 / q$ by (3.7). The theorem 
follows from 5.3 .

5.6. Quasiares. Suppose that $\alpha$ is a $(q, 2)$ thick $K$-quasiarc in $R^{2}$. Then there is a $K-Q C$ map $f: \dot{R}^{2} \rightarrow \dot{R}^{2}$ such that $f \alpha$ is a line segment. If $f(\infty)=\infty$, then $f$ is $s$-QS with $s=s(K) \rightarrow 0$ as $K \rightarrow 1$ by 2.13. By Theorem 5.5, we have $q$ $\leq \sqrt{11 s}$. For small $K, 2.13$ gives

$$
q \leq \sqrt{77} \sqrt[8]{K-1}
$$

One can show that this is also true if $f(\infty) \neq \infty$. However, we give a better bound in 5.9 below using directly the quasiconformality of $f$. We need the following result on the distortion of $\mathrm{QC}$ maps of the plane:

5.7. Lemma. Suppose that $f: R^{2} \rightarrow R^{2}$ is a $K-\mathrm{QC}$ map with $f(0)=0$, $f\left(e_{1}\right)=e_{1}, K \leq 2$. Then for each $x \in\left[0, e_{1}\right]$ we have

$$
d\left(f x,\left[0, e_{1}\right]\right) \leq \frac{1}{2} \sqrt{G(K)^{2}-1} \leq 4 \sqrt{K-1},
$$

where $G(K)$ is defined by $1 / G(K)=2 \varphi_{K, 2}(1 / \sqrt{2}) \varphi_{1 / K, 2}(1 / \sqrt{2})$, and $\varphi_{K, 2}$ is as in [Vu].

Proof. By [Vu], Cor. 3.15 (see also [AG], Th. 2), we have

$$
|f x|+\left|f x-e_{1}\right| \leq G(K) \leq 8^{K-1} \text {. }
$$

Hence $f x$ is located in the ellipse with foci 0 and $e_{1}$ and with focal sum $G(K)$. Thus $d\left(f x,\left[0, e_{1}\right]\right) \leq b$ where $2 b=\left(G(K)^{2}-1\right)^{1 / 2}$ is the smaller axis of this ellipse. We have

$$
4 b^{2}=64^{K-1}-1 .
$$

Since the expression $\left(a^{x}-1\right) / x$ is increasing in $x$ for $a>1, x>0$, and since $K \leq 2$, we obtain

$$
4 b^{2} \leq 63(K-1)
$$

and the lemma follows.

5.8. Remark. If we apply the results from [VV], we can slightly improve the upper bound in Lemma 5.7. 
5.9. THEOREM. Suppose that $\alpha$ is a $(q, 2)$-thick $K$-quasiarc in $R^{2}$. Then $q \leq 8 \sqrt{K-1}$.

Proof. Since we always have $q \leq 3 \sqrt{3} / 4$ by (4.2), we may assume that $K \leq 2$. Choose a $K$-QC map $f: \dot{R}^{2} \rightarrow \dot{R}^{2}$ with $f a=\left[0, e_{1}\right]$. Let $a$ be the endpoint of $\alpha$ with $f a=0$. We first treat the case $f(\infty) \neq \infty$, and set $R=\left|a-f^{-1}(\infty)\right|$. Choose a positive number $t<1$ such that $\alpha \backslash \bar{B}(a, t R) \neq \emptyset$. Since $\alpha$ is $(q, 2)$-thick, there is a 2 -simplex $\Delta$ with

$$
\Delta^{0} \subset \alpha \cap \bar{B}(a, t R), \quad m(\Delta) \geq q t^{2} R^{2} .
$$

There is $K_{1}=K_{1}(t)$ and a $K_{1}-\mathrm{QC}$ map $g: \dot{R}^{2} \rightarrow \dot{R}^{2}$ such that $g(\infty)=f^{-1}(\infty)$, $g \mid B(a, t R)=\mathrm{id}$, and $K_{1}(t) \rightarrow 1$ as $t \rightarrow 0$. The map $f_{1}=f g: \dot{R}^{2} \rightarrow \dot{R}^{2}$ is $K_{1} K-\mathrm{QC}, f_{1}(\infty)=\infty$, and $f_{1}=f$ in $B(a, t R)$. Using auxiliary similarities and Lemma 5.7 we see that

$$
m(\Delta) \leq 8 t^{2} R^{2} \sqrt{K_{1} K-1}
$$

By (5.10) this implies

$$
q \leq 8 \sqrt{K_{1} K-1}
$$

Letting $t \rightarrow 0$ we obtain $q \leq 8 \sqrt{K-1}$. If $f(\infty)=\infty$, the argument is simpler, because the auxiliary map $g$ is not needed. We choose $r>0$ such that $\alpha \backslash \bar{B}(a, r)$ $\neq \emptyset$ and choose $\Delta$ as above with $t R$ replaced by $r$.

5.11. Example. For $K \geq 1$ let $q_{1}(K)$ be the least upper bound of all $q>0$ such that there is a $(q, 2)$-thick $K$-quasiarc in $R^{2}$. By 5.9 we have

$$
q_{1}(K) \leq 8 \sqrt{K-1} .
$$

We next show that for $K \leq 9 / 4$ we have

$$
q_{1}(K) \geq \frac{\sqrt{K}-1}{768} \geq \frac{K-1}{1920}
$$

by constructing a $K-\mathrm{QC}$ map $f: R^{2} \rightarrow R^{2}$ which maps the segment $\left[-e_{1}, e_{1}\right]$ onto a $(q, 2)$-thick arc with $q=(\sqrt{K}-1) / 768$.

The map $f$ will be a limit of piecewise linear $K-\mathrm{QC}$ maps $f_{j}: R^{2} \rightarrow R^{2}$. Let $f_{0}$ be the identity map. We next construct $f_{1}$. 


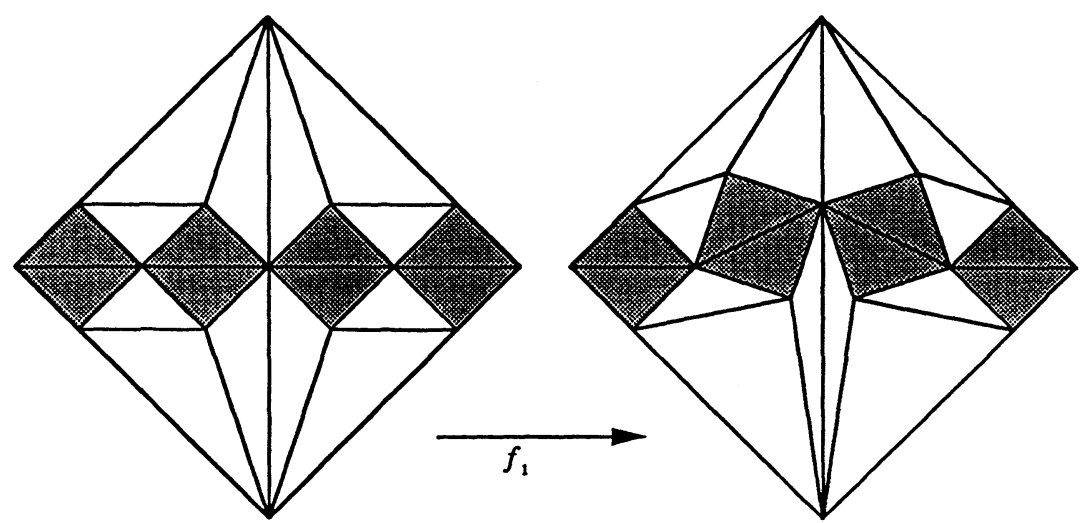

Figure 1

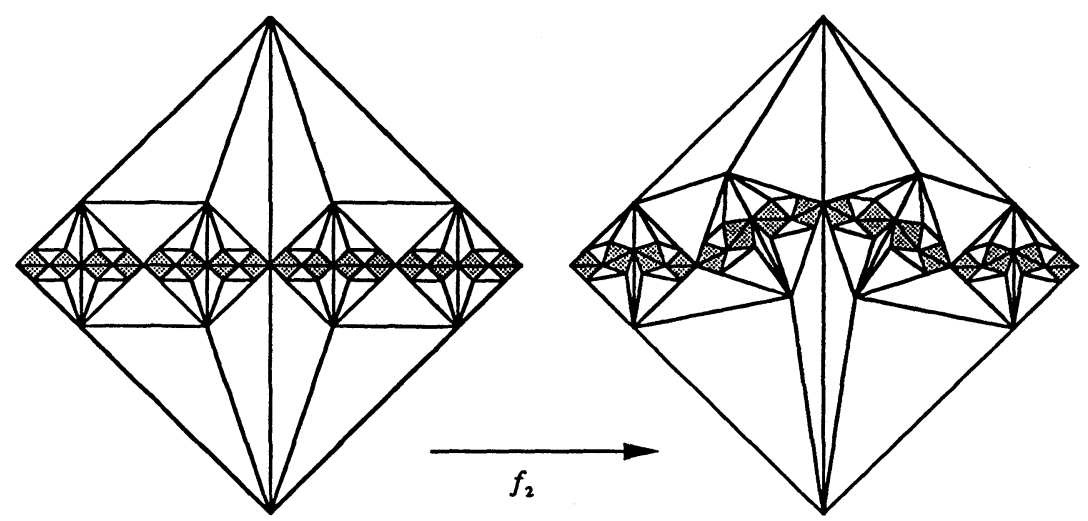

Figure 2

Let $Q_{0}$ be the closed square with vertices $\pm e_{1}$, $\pm e_{2}$. Let $0<t<1 / 24$, and let $f_{1}: Q_{0} \rightarrow Q_{0}$ be the simplicial homeomorphism described by Figure 1: the point $f_{1}(0)$ is chosen to be $t e_{2}$. The four shaded areas $Q_{1}, Q_{2}, Q_{3}, Q_{4}$ are squares of diameter $1 / 2$, and each $f_{1} \mid Q_{i}$ is a similarity. Indeed, $f_{1} \mid Q_{1} \cup Q_{4}=$ id. Since $f_{1} \mid \partial Q_{0}$ $=$ id, we can extend $f_{1}$ by the identity to a homeomorphism $f_{1}: R^{2} \rightarrow R^{2}$. The map $f_{1}$ is $K-\mathrm{QC}$ with $K=K(t)$, which will be computed later.

The map $f_{2}$ is described in Figure 2. Outside the four squares $Q_{i}$ we have $f_{2}=f_{1}$. In $Q_{i}, f_{2}$ is of the form $\beta_{i} f_{1} \alpha_{i}$, where $\alpha_{i}: Q_{i} \rightarrow Q_{0}$ and $\beta_{i}: Q_{0} \rightarrow f_{1} Q_{i}$ are similarities. The map $f_{2}$ is $K-\mathrm{QC}$ with the same $K$ as $f_{1}$.

Proceeding inductively we obtain a sequence of $K-\mathrm{QC}$ maps $f_{j}: R^{2} \rightarrow R^{2}$. The map $f_{j+1}$ agrees with $f_{j}$ outside $4^{j}$ squares of diameter $2 \cdot 4^{-j}$, and $f_{j}$ is a similarity in these squares. The maps $f_{j}$ clearly converge to a $K$-QC map $f: R^{2} \rightarrow R^{2}$. Thus 
$\alpha=f\left[-e_{1}, e_{1}\right]$ is a $K$-quasiarc.

We show that $\alpha$ is $(q, 2)$-thick with $q=t / 64$. Let $x \in \alpha$ and $r>0$ be such that $\alpha \backslash \bar{B}(x, r) \neq \emptyset$. In the $j^{\text {th }}$ step of the construction, $x$ belongs to the $f_{j}$-image of one the $4^{j}$ squares of this step; call this image $R_{j}$. Then $Q_{0}=R_{0} \supset R_{1} \supset \ldots$ and $d\left(R_{j}\right) \geq d\left(R_{j-1}\right) / 4$. Let $j$ be the smallest integer with $R_{j} \subset \bar{B}(x, r)$, and set $u_{j}=d\left(R_{j}\right)$. Since $\alpha \subset Q_{0}=R_{0}$, we have $j \geq 1$. Moreover, $x \in R_{j-1} \not \subset \bar{B}(x, r)$, which implies that $u_{j-1} \geq r$, and hence

$$
u_{j} \geq r / 4 \text {. }
$$

The square $R_{j}$ has two opposite vertices $a_{0}, a_{1}$ in $\alpha$. There is also a point $a_{2} \in \alpha$ such that the 2-simplex $a_{0} a_{1} a_{2}$ is similar to the 2 -simplex with vertices $e_{1},-e_{1}$, $t e_{2}$. Thus

$$
m(\Delta)=t u_{j}^{2} / 4 \geq t r^{2} / 64 .
$$

Since $\Delta \subset R_{j} \subset \bar{B}(x, y), \alpha$ is $q$-thick with $q=t / 64$.

We next show that $f$ is $K-\mathrm{QC}$ with $K=(1+12 t)^{2}$. Consider the basic triangulation $T$ of $Q_{0}$ given in Figure 1 . If $v$ is a vertex of $T$, then $f_{1} v \neq v$ only for $v=$ 0 and for the four vertices $v=\left( \pm e_{1} \pm e_{2}\right) / 4$. It is easy to see that for these $v$ we have $\left|f_{1} v-v\right| \leq t$. If $\Delta$ is a 2 -simplex of $T, f_{1}$ keeps at least one vertex of $\Delta$ fixed. The smallest height $b(\Delta)$ of $\Delta$ is at least $1 / 4$. By Lemma 5.14 below, $f_{1} \mid \Delta$ is $(1+12 t)$-bilipschitz. Hence $f_{1}: R^{2} \rightarrow R^{2}$ is $(1+12 t)$-bilipschitz and hence $(1+12 t)^{2}-\mathrm{QC}$. Hence we can choose $K=(1+12 t)^{2}$.

It follows that

$$
q=\frac{t}{64}=\frac{\sqrt{K}-1}{768}
$$

and we have proved (5.13). It is possible to compute the dilatation of $f_{1}$ exactly, but this would only replace the constant 768 by a smaller one, and there would still remain an essential difference between the bounds (5.12) and (5.13).

5.14. Lemma. Suppose that $\Delta=a_{0} a_{1} a_{2}$ is a 2 -simplex in $R^{2}$ and that $f: R^{2}$ $\rightarrow R^{2}$ is an affine map such that

$$
f a_{0}=a_{0}, \quad\left|f a_{j}-a_{j}\right| \leq \alpha b(\Delta) / 3
$$

for $j=1,2$, where $0 \leq \alpha \leq 1 / 2$. Then $f$ is $(1+\alpha)$-bilipschitz.

Proof. In $\left[\mathrm{TV}_{2}\right]$, Lemma 3.5 , one proved that $f$ is $(1+2 \alpha)$-bilipschitz without the condition $f a_{0}=a_{0}$. The proof of the present lemma is obtained by a slight modification. 


\section{The bilipschitz case}

6.1. Bilipschitz maps. A map $f: X \rightarrow Y$ between metric spaces is called $M$-bilipschitz, $M \geq 1$, if

$$
|x-y| / M \leq|f x-f y| \leq M|x-y|
$$

for all $x, y \in X$. An $M$-bilipschitz map is $\eta$-QS with $\eta(t)=M^{2} t$ and hence $s-\mathrm{QS}$ with

$$
\begin{aligned}
& s=\left(M^{2}-1\right)^{1 / 2} \text { for } 1 \leq M \leq \sqrt{2}, \\
& s=M^{2}-1 \text { for } M \leq \sqrt{2} .
\end{aligned}
$$

In particular, $s \rightarrow 0$ as $M \rightarrow 1$. Hence the preceding sections give estimates for the change of the thickness of a set under a $M$-bilipschitz map. Hewever, using directly the bilipschitz condition we can easily obtain better results.

We start with an example which shows that thickness is not a bilipschitz invariant.

6.2. ExAmple. We shall construct a bilipschitz map $f: R^{2} \rightarrow R^{2}$ which carries a subset $A$ of the $x_{1}$-axis onto a set which is 2 -thick. Observe that $A$ cannot be an interval, since then $f A$ would be a rectifiable arc and hence not 2 -thick.

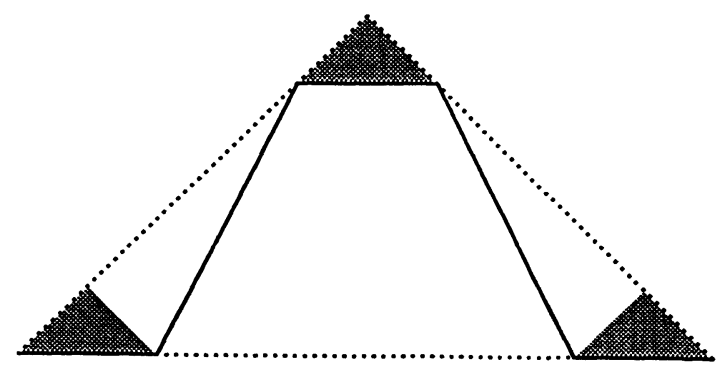

Figure 3

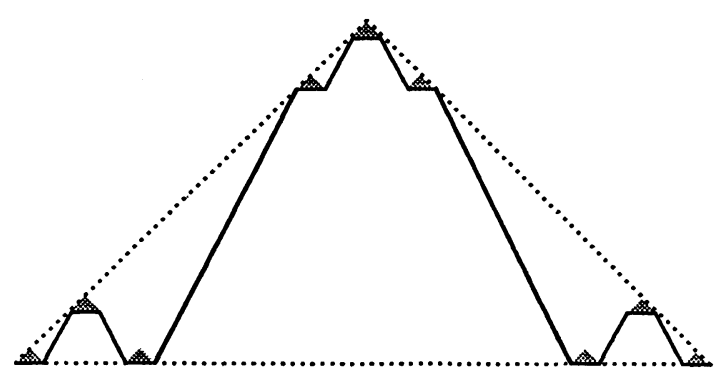

Figure 4 
Let $0<t \leq \sqrt{3} / 2$. We define a sequence of continuous real-valued functions $\varphi_{j}$ on $I=[0,1]$ as follows: Divide $I$ into successive intervals $I_{1}, \ldots, I_{5}$ of length $1 / 5$. Then $\varphi_{1}(x)=0$ for $x \in I_{1} \cup I_{5}$, and $\varphi_{1}(x)=4 / 5$ for $x \in I_{3}$. In the intervals $I_{2}$ and $I_{4} \varphi_{1}$ is affine. Then $\varphi_{1}: I \rightarrow R^{1}$ is $4 t$-Lipschitz. See Figure 3 , where $t=1 / 2$. The function $\varphi_{2}$ agrees with $\varphi_{1}$ on $I_{2} \cup I_{4}$. For $i=1,3,5$, the graph of $\varphi_{2} \mid I_{i}$ is similar to the graph of $\varphi_{1}$. For example, $\varphi_{2}(x)=\varphi_{1}(5 x) / 5$ for $x \in I_{1}$. The function $\varphi_{2}$ is also $4 t$-Lipschitz. See Figure 4 .

Proceeding similarly we obtain an increasing sequence of $4 t$-Lipschitz functions $\varphi_{j}$ converging to a $4 t$-Lipschitz function $\varphi: I \rightarrow R^{1}$. The function $\varphi_{j}$ is constant on $3^{j}$ closed intervals of length $5^{-j}$; let $A_{j}$ be the union of these intervals. Then the intersection of all $A_{j}$ is a Cantor set $A \subset I$. The graph $C$ of $\varphi \mid A$ is a self-similar Cantor set in $R^{2}$. It can be obtained from the triangle $\Delta_{0}$ with vertices $0, e_{1}, e_{1} / 2+t e_{2}$ by first replacing $\Delta_{0}$ by the three shaded triangles of Figure 3 and repeating this process infinitely.

Extend $\varphi$ to $R^{1}$ by setting $\varphi(x)=0$ for $x \in R^{1} \backslash I$. Then $\varphi$ is $4 t$-Lipschitz on $R^{1}$. Define $f: R^{2} \rightarrow R^{2}$ by

$$
f(x, y)=(x, y+\varphi(x))=(x, y)+\varphi(x) e_{2} .
$$

Then $f$ is $(1+4 t)$-Lipschitz. Since $f$ is a homeomorphism with $f^{-1}(x, y)=$ $(x, y-\varphi(x))$, we similarly see that $f$ is in fact $(1+4 t)$-bilipschitz. Furthermore, $f$ maps the set $A$ in the real axis onto $C$.

We show that $C$ is $(q, 2)$-thick with $q=t / 50$. Suppose that $x \in C$ and $r>0$ with $C \backslash \bar{B}(x, r) \neq \emptyset$. Since $d(C)=1$, we have $r<1$. Choose an integer $j$ with $5^{-j}<r \leq 5^{-j+1}$; then $j \geq 1$. In the $j^{\text {th }}$ step of the construction, $x$ belongs to a triangle $\Delta$ such that $d(\Delta)=5^{-j}, \Delta^{0} \subset C$, and $\Delta$ is similar to $\Delta_{0}$. Thus $m(\Delta)=$ $5^{-2 j} t / 2$. Since $r \leq 5^{-j+1}$, we have $m(\Delta) \geq r^{2} t / 50$. Clearly $\Delta \subset \bar{B}(x, r)$, and hence $C$ is $(q, 2)$-thick with $q=t / 50$.

We have proved that if $1<M \leq 1+2 \sqrt{3}$, then there is an $M$-bilipschitz homeomorphism $f: R^{2} \rightarrow R^{2}$ such that $f$ carries a subset of the real axis onto a $(q, 2)$-thick set with $q=(M-1) / 200$.

Observe the striking difference between bilipschitz homeomorphisms and diffeomorphisms of $R^{2}$. In a diffeomorphism $h: R^{2} \rightarrow R^{2}$, the curve $h R^{1}$ has a tangent at every point and cannot contain any 2 -thick set.

Let $g: A \rightarrow C$ be the homeomorphism defined by $f$. Of course, $g$ is also ( $1+$ $4 t$ )-bilipschitz. However, we show that $g$ is in fact $\left(1+8 t^{2}\right)$-bilipschitz. The map $g^{-1}: C \rightarrow A$ is the restriction of the orthogonal projection and hence 1-Lipschitz. On the other hand, if $x, y \in C$, then $\left|x_{2}-y_{2}\right| \leq 4 t\left|x_{1}-y_{1}\right|$. From this it follows that 


$$
|x-y|^{2} \leq\left(1+16 t^{2}\right)\left|x_{1}-y_{1}\right|^{2} \leq\left(1+8 t^{2}\right)^{2}\left|x_{1}-y_{1}\right|^{2} \text {, }
$$

which implies that $g$ is $\left(1+8 t^{2}\right)$-Lipschitz. Hence, for every $M \leq 7$ there is an $M$-bilipschitz map $g: A \rightarrow R^{2}$ such that $g A$ is $(q, 2)$-thick with $q=\sqrt{M-1}$ $/(50 \sqrt{8})>\sqrt{M-1} / 142$.

6.3. Bilipschitz theory. We shall give bilipschitz versions of parts of Sections 3 and 4 . Corresponding to 3.12 , we first consider a $k$-simplex $\Delta=a_{0} \ldots a_{k}$ $\subset E$ with $\rho(\Delta) \leq r$ and an $M$-bilipschitz map $f: \Delta^{0} \rightarrow E^{\prime}$. With the notation of 3.12 we then have the inequalities

$$
M^{-1} d_{i j} \leq d_{i j}^{\prime} \leq M d_{i j}
$$

which replace (1) of 3.12 . The proof of 3.12 then gives

$$
\begin{aligned}
& |\Gamma(f \bar{a})| \geq \lambda(M, r, k)|\Gamma(\bar{a})|, \\
& \lambda(M, r, k)=M^{-2 k}-2^{-k-1}(k+2) ! r^{k(k+1)}\left(M^{2 k}-M^{-2 k}\right) .
\end{aligned}
$$

Thus $\lambda(M, r, k) \rightarrow 1$ as $M \rightarrow 1$ and $\lambda(M, r, k)>0$ for $1 \leq M \leq M^{*}$ with some $M^{*}=M^{*}(r, k)$.

Corresponding to 4.3 , we next suppose that $A \subset E$ is $(q, k)$-thick and that $f: A \rightarrow E^{\prime}$ is $M$-bilipschitz, $M>1$. We want to find a lower bound for the thickness of $f A$. Let $y_{0}=f\left(x_{0}\right) \in f A$ and let $r>0$ with $f A \backslash \bar{B}\left(y_{0}, r\right)=F^{\prime} \neq \emptyset$. Writing $F=f^{-1} F^{\prime}$ and $r_{1}=d\left(F, x_{0}\right)$ we have $r_{1} \geq r / M$. Set $r_{2}=r_{1} / M$ and choose a $k$-simplex $\Delta=a_{0} \ldots a_{k}$ such that $a_{j} \in A \cap \bar{B}\left(x_{0}, r_{2}\right)$ and $m_{k}(\Delta) \geq$ $q r_{2}^{k}$. Writing $a_{j}^{\prime}=f a_{j}$ and $\Delta^{\prime}=a_{0}^{\prime} \ldots a_{k}^{\prime}$ we have $\left|a_{j}^{\prime}-y_{0}\right| \leq r$. As in 4.3 we obtain $\rho(\Delta) \leq c_{k} / q$ with the same constant $c_{k}$. Using (6.4) we get $m_{k}\left(\Delta^{\prime}\right) \geq q_{1} r^{k}$, where

$$
q_{1}=q_{1}(q, M, k)=q M^{-2 k} \lambda\left(M, c_{k} / q, k\right)^{1 / 2} .
$$

This function is positive for $1 \leq M \leq M^{*}\left(c_{k} / q, k\right)=M_{0}(q, k)$. We have proved the following bilipschitz version of Theorem 4.3:

6.5. THEOREM. Let $k$ be a positive integer and let $q>0$. There is a number $M_{0}=M_{0}(q, k)>1$ and a positive decreasing function $M \mapsto q_{1}(q, M, k)$, defined on $\left[1, M_{0}\right]$, such that:

(1) $\lim _{M \rightarrow 1} q_{1}(q, M, k)=q$.

(2) If $A \subset E$ is $(q, k)$-thick and if $f: A \rightarrow E^{\prime}$ is $M$-bilipschitz, $M \leq M_{0}$, then $f A$ is $\left(q_{1}, k\right)$-thick.

The bounds are explicit. 
6.6. A special problem. We consider $M$-bilipschitz maps $f: A \rightarrow E$ where $A \subset R^{1}$ and $E$ is an arbitrary inner product space. Let $q^{*}(M)$ be the supremum of all $q$ such that $f A$ is $(q, 2)$-thick for some $f$ and $A$. The example in 6.2 shows that

$$
q^{*}(M) \geq \frac{\sqrt{M-1}}{142}
$$

for $M \leq 7$. We show that this estimate has the correct order of magnitude by proving that

$$
q^{*}(M) \leq 3 \sqrt{M-1}
$$

for all $M \geq 1$. Since (4.2) gives $q^{*}(M) \leq 3 \sqrt{3} / 4$ for all $M$, we may assume that $M \leq 5 / 4$.

Suppose that $A \subset R^{1}$, that $f: A \rightarrow E$ is an $M$-bilipschitz map and that $f A$ is $(q, 2)$-thick. We must show that $q \leq 3 \sqrt{M-1}$. Choose $x \in f A$ and $r>0$ such that $f A \backslash \bar{B}(x, r) \neq \emptyset$. Since $f A$ is $(q, 2)$-thick, we can choose points $a<b<c$ in $A$ such that $\Delta=f(a) f(b) f(c)$ is a triangle with $m(\Delta) \geq q r^{2}$. Write $\alpha=$ $|f a-f c|$, and let $h$ be the height of $\Delta$ from $f b$. The bilipschitz condition gives

$$
|f a-f b|+|f b-f c| \leq M|a-b|+M|b-c|=M|a-c| \leq M^{2} \alpha .
$$

Let $T$ be the 2-dimensional plane containing $\Delta$. Then $f b$ is within the ellipse of $T$ with foci $f a$ and $f c$ and focal sum $M^{2} \alpha$. This obviously implies that

$$
4 h^{2} \leq M^{4} \alpha^{2}-\alpha^{2}=\alpha^{2}\left(M^{2}+1\right)(M+1)(M-1) \leq 6 \alpha^{2}(M-1) .
$$

Since $\alpha \leq 2 r$, we obtain

$$
q \leq \frac{m(\Delta)}{r^{2}}=\frac{\alpha h}{2 r^{2}} \leq \sqrt{6(M-1)} \leq 3 \sqrt{M-1}
$$

as desired.

\section{REFERENCES}

[AG] S. Agard and F. W. Gehring, Angles and quasiconformal mappings, Proc. London Math. Soc., 14A (1965), 1-21.

[AVV] G. D. Anderson, M. K. Vamanamurthy and M. Vuorinen, Inequalities for quasiconformal maps in space, Pacific Math. J., 160 (1993), 1-18.

[Be] M. Berger, Geometry I, Springer-Verlag, 1987.

[B1] L. M. Blumenthal, Theory and applications of distance geometry, Oxford at the Clarendon Press, 1953.

[Ha] H. Hadwiger, Vorlesungen über Inhalt, Oberfläche und Isoperimetrie, Springer- 
Verlag, 1957.

[JW] A. Jonsson and H. Wallin, Function spaces on subsets of $\mathbf{R}^{n}$, Harwood Acad. Publ., 1984.

[MV] P. Mattila and M. Vuorinen, Linear approximation property, Minkowski dimension and quasiconformal spheres, J. London Math. Soc., (2) 42 (1990), 249-269.

[NV] R. Näkki and J. Väisälä, John disks, Exposition. Math,. 9 (1991), 3-43.

$[\mathrm{Pa}] \mathrm{J}$. Partanen, Invariance theorems for the bilipschitz and quasisymmetric extension properties, Ann. Acad. Sci. Fenn. Ser. A I Math. Diss. 80 (1990), 1-40.

[Ri] S. Rickman, Characterization of quasiconformal arcs, Ann. Acad. Sci. Fenn. Ser. A I Math., 395 (1966), 1-30.

[Ro] S. Rohde, On conformal welding and quasicircles, Michigan Math. J., 38 (1991), $111-116$.

[So] D. M. Y. Sommerville, An introduction to the geometry of $n$ dimensions, Dover Publications, 1958.

[TV $\left.{ }_{1}\right]$ P. Tukia and J. Väisälä, Quasisymmetric embeddings of metric spaces, Ann. Acad. Sci. Fenn. Ser. A I Math., 5 (1980), 97-114.

$\left[\mathrm{TV}_{2}\right]$ - Extension of embeddings close to isometries or similarities, Ann. Acad. Sci. Fenn. Ser. A I Math., 9 (1984), 153-175.

[Vä1] J. Väisälä, Lectures on n-dimensional quasiconformal mappings, Lecture Notes in Mathematics, 229, Springer-Verlag, 1971.

[Vä2] - Quasimöbius maps, J. Analyse Math., 44, (1984/85), 218-234.

[Vä3] - Bilipschitz and quasisymmetric extension properties, Ann. Acad. Sci. Fenn. Ser. A I Math., 11 (1986), 239-274.

[Vä4] - Q Quasiconformal maps of cylindrical domains, Acta Math., 162 (1989), $201-225$.

[VV] M. K. Vamanamurthy and M. Vuorinen, Functional inequalities, Jacobi products, and quasiconformal maps, Illinois J. Math., 38 (1994), 394-419.

[Vu] M. Vuorinen, Quadruples and spatial quasiconformal mappings, Math. Z., 205 (1990), 617-628.

[WW] H. Wallin and P. Wingren, Dimension and geometry of sets defined by polynomial inequalities, J. Approx. Theory 69 (1992), 231-249.

J. Väisälä and M. Vuorinen:

Mathematiikan laitos

Helsingin yliopisto

Hallituskatu 15

FIN-00100 Helsinki

Finland

H. Wallin:

Matematiska institutionen

Umeå universitet

S-90187 Umeå

Sweden 